\title{
Correction to: Chronic Postoperative Pain After Robot-Assisted Laparoscopic Hysterectomy for Endometrial Cancer by Lunde S, Petersen KK, Kugathasan P, Arendt-Nielsen $L$ and Søgaard-Andersen E. Journal of Gynecologic Surgery 2019;35(3);140-146. DOI: 10.1089/gyn.2018.0068
}

$\mathbf{I}^{2}$

N THE JUNE 2019 issue of Journal of Gynecologic Surgery (vol. 35, no. 3; 140-146) an error was identified in Table 4 on page 144 where three out of the thirteen 95\% confidence intervals were incorrectly reported (Age in Model 1, Acute postoperative pain, and Age in Model 2). Please see below for the original Table 4 (left) and revised Table 4 (right) indicating the corrected data in bold.

TABle 4. Binary Logistic Regression With BackWard Stepwise Selection of Risk Factors For Postoperative Chronic Pain Following RoBot-Assisted HySTERECTOMY

Model 1 Nagelkerke $R^{2} 0.419$

\begin{tabular}{lcc}
\hline Variable & OR $(95 \% \text { CI })^{\mathrm{a}}$ & $\mathrm{p}$-Value \\
\hline Preoperative pelvic pain & $4.24(3.34-5.14)$ & 0.002 \\
Acute postoperative pain & $1.20(1.00-1.40)$ & 0.067 \\
Preoperative pain elsewhere & $2.66(1.52-3.80)$ & 0.092 \\
Age & $0.96(0.14-1.78)$ & 0.351 \\
Severity of cancer & $1.97(0.97-2.97)$ & 0.182 \\
Operating time & $1.00(0.98-1.02)$ & 0.896 \\
Blood loss & $1.01(0.99-1.03)$ & 0.290 \\
Education & $1.41(1.06-1.76)$ & 0.053 \\
Employment status & $0.94(0.63-1.25)$ & 0.695 \\
\hline
\end{tabular}

Model 2 Nagelkerke $R^{2} 0.382$

\begin{tabular}{llr}
\hline Variable & \multicolumn{1}{c}{ OR $(95 \% \text { CI })^{\mathrm{a}}$} & $\mathrm{p}$-Value \\
\hline Preoperative pelvic pain & $4.99(4.15-5.83)$ & 0.000 \\
Acute postoperative pain & $1.27(-0.55$ to 3.09$)$ & 0.009 \\
Age & $0.94(0.31-1.57)$ & 0.056 \\
Education & $1.34(1.01-1.67)$ & 0.074 \\
\hline
\end{tabular}

${ }^{\mathrm{a}}$ Data are displayed as OR $(95 \% \mathrm{CI})$.

OR, odds ratio; CI, confidence interval.
TABLE 4. BinARY Logistic REgRESSION With BackWard Stepwise Selection of Risk Factors for Postoperative Chronic Pain Following RoBot-Assisted Hysterectomy

Model 1 Nagelkerke $R^{2} 0.419$

\begin{tabular}{lcc}
\hline Variable & OR $(95 \% \text { CI })^{\mathrm{a}}$ & $\mathrm{p}$-Value \\
\hline Preoperative pelvic pain & $4.24(3.34-5.14)$ & 0.002 \\
Acute postoperative pain & $1.20(1.00-1.40)$ & 0.067 \\
Preoperative pain elsewhere & $2.66(1.52-3.80)$ & 0.092 \\
Age & $0.96(\mathbf{0 . 8 8}-\mathbf{1 . 0 4})$ & 0.351 \\
Severity of cancer & $1.97(0.97-2.97)$ & 0.182 \\
Operating time & $1.00(0.98-1.02)$ & 0.896 \\
Blood loss & $1.01(0.99-1.03)$ & 0.290 \\
Education & $1.41(1.06-1.76)$ & 0.053 \\
Employment status & $0.94(0.63-1.25)$ & 0.695 \\
\hline
\end{tabular}

Model 2 Nagelkerke $R^{2} 0.382$

\begin{tabular}{lcc}
\hline Variable & OR $(95 \% \text { CI })^{\mathrm{a}}$ & $\mathrm{p}$-Value \\
\hline Preoperative pelvic pain & $4.99(4.15-5.83)$ & 0.000 \\
Acute postoperative pain & $1.27(\mathbf{1 . 0 9 - 1 . 4 5 )}$ & 0.009 \\
Age & $0.94(\mathbf{0 . 8 8}-\mathbf{1 . 0 0})$ & 0.056 \\
Education & $1.34(1.01-1.67)$ & 0.074 \\
\hline
\end{tabular}

${ }^{\mathrm{a}}$ Data are displayed as OR $(95 \% \mathrm{CI})$.

OR, odds ratio; $\mathrm{CI}$, confidence interval. 
Subsequently, the article refers to two of the three incorrect $95 \%$ confidence intervals in the section "Risk factors for development of chronic postoperative pain" on page 143. See below for the original text as well as the revised text with corrected data indicated in bold.

\section{Risk factors for development of chronic postoperative pain}

The binary logistic regression model with a backward stepwise selection of risk factors was made in 2 models. Model 1 (Nagelkerke $\mathrm{R}^{2}$ 0.419) included all study parameters (preoperative pelvic pain, acute postoperative pain intensity, preoperative pain elsewhere, age, severity of cancer stage \pm lymphadenectomy, operating time, blood loss, education, and employment status). Model 2 (Nagelkerke $\mathrm{R}^{2}$ 0.382) only included 4 study parameters from model 1 (preoperative pelvic pain, acute postoperative pain intensity, age, and education). The regression model established 2 significant factors for development of chronic postoperative pain $(p<0.05)$ : preoperative pelvic pain (odds ratio [OR]: $4.99,95 \% \mathrm{CI}: 4.15-5.83)$ and acute postoperative pain (OR: 1.27 ; $95 \%$ CI -0.55 to 3.09 ) while demonstrating a trend toward age as a protective factor (OR: 0.94: 95\% CI: 0.31-1.57). Please refer to Table 4.

The authors apologize for this error.

The online version of this article has been corrected.

\section{Risk factors for development of chronic postoperative pain}

The binary logistic regression model with a backward stepwise selection of risk factors was made in 2 models. Model 1 (Nagelkerke $\mathrm{R}^{2}$ 0.419) included all study parameters (preoperative pelvic pain, acute postoperative pain intensity, preoperative pain elsewhere, age, severity of cancer stage \pm lymphadenectomy, operating time, blood loss, education, and employment status). Model $2(\mathrm{Na}-$ gelkerke $\mathrm{R}^{2}$ 0.382) only included 4 study parameters from model 1 (preoperative pelvic pain, acute postoperative pain intensity, age, and education). The regression model established 2 significant factors for development of chronic postoperative pain $(p<0.05)$ : preoperative pelvic pain (odds ratio [OR]: $4.99,95 \% \mathrm{CI}: 4.15-5.83$ ) and acute postoperative pain (OR: 1.27; 95\% CI: 1.09-1.45) while demonstrating a trend toward age as a protective factor (OR: 0.94: $95 \% \mathrm{CI}$ : 0.88-1.00). Please refer to Table 4. 